\title{
GESTIÓN DEL RIESGO EN CASO DE INCENDIO EN EL HOSPITAL NACIONAL DE NIÑOS, COSTA RICA
}

\author{
Adriana Flores \& Rocío Valverde (2011)
}

En este trabajo final de investigación aplicada, se elaboró un plan de acción en Gestión del Riesgo en caso de incendio en las instalaciones del Hospital Nacional de Niños, único hospital pediátrico en Costa Rica.

El impacto de la aplicación de este plan, tendrá un efecto a nivel nacional y podría ser utilizado como referencia por otros hospitales del país adaptándolo a su realidad. Máxime que a nivel nacional aun no se ha publicado un estudio similar, en el cuál el riesgo de incendio sea abordado desde una perspectiva integral, tomando en cuenta la opinión de los directivos del centro hospitalario.

Se utilizó una metodología cualitativa y se aplicó el análisis de vulnerabilidades y capacidades (ACV) que utiliza la Federación Internacional de Sociedades de la Cruz Roja y Media Luna Roja, la cual se complementó con la fórmula de la Universidad de Wisconsin-Madison para la evaluación de la amenaza. En el proceso de investigación participaron miembros del Consejo de Dirección del hospital a través de entrevistas, para identificar la amenaza de incendio, así como la vulnerabilidad interna. También se realizaron talleres con el Comité de Emergencias Local y los Coordinadores de Brigada, donde se estimulo la sensibilización de los participantes sobre el riesgo de que ocurra un incendio hasta llegar a generar acciones para fomentar la resiliencia (capacidad para adaptarse o resistir).

A partir del trabajo en equipo se determinaron los elementos y factores que pueden dar origen a un incendio y se identificaron las instalaciones más vulnerables del hospital, así mismo se analizó la capacidad para enfrentar los casos de incendio.

Se concluye que este hospital ha cumplido con la capacitación a los funcionarios para la atención de emergencias por incendio, pero es necesario capacitar a los tomadores de decisión en materia de Gestión de Riesgo para que se involucren en el cambio más proactivo hacia la prevención.

Finalmente, con la información recopilada se propone un Plan de Acción en Gestión de Riesgo en Caso de Incendio que incluye diez proyectos, con actividades, responsables, plazos, financiamiento y forma de divulgación. 